\title{
Novel non-invasive Pressure-Volume Loop measurement for local Pulse Wave Velocity estimation
}

\author{
G.M.W. Janjua, D.D. Finlay, D. Guldenring, R. Hadia, J. McLaughlin \\ Ulster University, Belfast, United Kingdom
}

\begin{abstract}
In the last four decades, hypertension doubled to 1.13 billion patients. High blood pressure (BP) is the main risk factor for cardiovascular morbidity and mortality. Arterial stiffness (AS) is a key component and poorly understood part of cardiac vital signs. Pressure-volume loop (PULoop) has been used to measure local pulse wave velocity $(P W V)$ which is an indicator of AS [1].

We have been able to measure the PU-Loop noninvasively on palmar digital arteries. Pressure and flow waveforms are measured simultaneously at the same location. The dataset has calculated the normalized PWV of $1.48 \pm 0.4$ from the slope of the line formed between two early systole points of 20-30\%. PU-Loop provides an insight into contractility, preload, and hypertension and correcting factor for pulse transit time estimations.
\end{abstract}

\section{Introduction}

Since the beginning of the $19^{\text {th }}$ century, cuff-based blood pressure (BP) measurement devices have been used in clinical practice and are widely used for hypertension assessment [2]. The blood pressure changes with every heartbeat and these snapshot measurements often lead to wrong diagnoses due to phenomenon's like white collar hypertension, depression etc. Brachial BP is reported to be higher than central BP and this can greatly affect the therapeutic treatment [2]. Hypertensive treatment drugs show different pharmacological responses on dropping BP which are sometimes only notable in central BP.

Arterial stiffness plays a vital role in cardiovascular diseases and is a primary cause of high BP. Arterial stiffness complicates the situation in hypertensive subjects which lead to different BP in vital organs such as the brain, kidneys etc. due to various levels of wave reflection and their arrival times.

Global PWV is measured over longer distances on the arterial tree, most commonly measured from carotid to femoral (C2F). It gives average PWV due to mixed composition of the elastic and muscular material of aortic artery. Local PWV is measured over a short segment of the artery and showed enormous potential for vascular diseases [3]. Pulse transit time (PTT) relation to BP has been studied over the last decade and showed valid and well-accepted affiliation [4].

PWV can be used to measure the arterial stiffness which in turn shows good co-relation to BP [5]. Invasive pressure-volume (PU) loop, the gold standard, has been used for measuring cardiac functionality during the heart's working cycle. In invasive PU loop, a conductance catheter is inserted into left ventricle which simultaneously measures the chamber's blood pressure and volume waveforms at same location [6]. This three-decade-old technique measures ventricular compliance and contractility by measuring changes in afterload and preload during the cardiac cycle [7].

We are presenting a novel non-invasive PU loop measurement method for e-Health applications that will ultimately allow for early detection of cardiovascular diseases (CVD) in hypertensive patients.

\section{Background}

The cardiac cycle is a closed-loop system and the PU loop consists of four stages, ventricular filling, isovolumetric contraction, ejection and isovolumetric relaxation which in turn produces systole and diastole BP. Left ventricle and aorta interface at aortic valve plays a pivotal relation to cardiovascular dysfunction. The capacity of the aorta, arterial compliance, to retain the amount of blood ejected by left ventricle plays a vital role in the blood circulation system. Lower arterial compliance results in higher BP and higher load on the left ventricle.

Due to higher risk in the invasive procedure noninvasive methods have been developed which are easier to use on a larger number of subjects. Echocardiogram (ECG) gated non-invasive PU loop measurement is becoming more common in clinical practice due to less risk during assessment [8]. The most famous method for non-invasive PU loop analysis is 1) 2D \& 3D Echocardiogram, 2) Tissue Doppler imaging (TDI), 3) phase-contrast Medical Resonance Imaging and 4) Micro-computed tomography (micro-CT) [9]. These image analysis modalities have produced useful results over the last decade. Due to the complexity of analysis and limitation in biomechanical 
properties no wearable approach is feasible only ultrasound based systems are used to measure local PWV in a variety of arteries [10].

\section{Materials and methods}

For this experiment, we have recruited five healthy and one borderline hypertensive volunteers of the age of $32 \pm 6.5$ years (all males) and vital sign dataset for 120 seconds was collated. After relaxing for 5 minutes in a seated position on an armchair, we have collected the ECG, Photoplethysmogram (PPG) and Ballistic Pressure pulse (BPP) signals to measure the electrical, optical and mechanical activity of blood flow from the heart to distal arteries. The pressure waveform is measured by BPP sensor and the flow waveform is measured PPG sensor.

\subsection{Apparatus}

Biopac MP36R system (fig. 2) is used for simultaneous sensor data acquisition - For our experiment sensors used are: 1) to measure cardiac activity, we have placed 2-lead ECG sensor on subject's chest for stable anchoring position for noise and artifact-free signals, 2) to measure blood flow waveform, a PPG sensor of $960 \mathrm{~nm}$ wavelength is used on subject's digital palmar artery in index finger, and 3) to measure pulse pressure waveform, a poor man tonometer system is designed which uses Measurement Specialties polyvinylidene fluoride (PVDF) piezoelectric sensor [10] to measure distension waveform of artery results due to pulsating blood flow. The sensor is strappedup around the finger which occludes the subject's palmar artery in index finger against the bone eventually enables the sensor to measure the ballistic force on artery surface by blood pulsation. We called this sensor as ballistic pulse pressure (BPP) sensor. The piezoelectric sensor is interfaced to Film Lab which is digitized by Biopac analog input. Amplifier settings are shown in table 1.

Table 1: Piezo Film Lab amplifier settings

\begin{tabular}{cc}
\hline Mode & Voltage $-1 \mathrm{G}(50 \mathrm{pF})$ \\
\hline Filter low frequency & $0.1 \mathrm{~Hz}$ \\
\hline Filter high frequency & $10 \mathrm{~Hz}$ \\
\hline Gain & $10 \mathrm{~dB}$ typical \\
\hline Power source & $9 \mathrm{~V}$ Battery \\
\hline
\end{tabular}

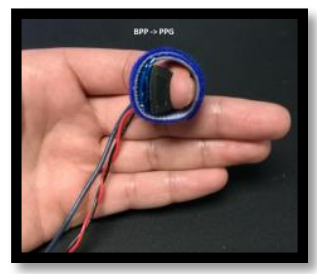

Figure 1: BPP and PPG stack-aligned sensor placement

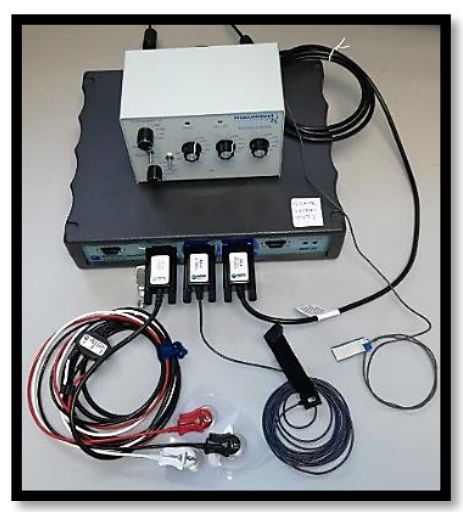

Figure 2: ECG sensor (Left), PPG Sensor (Center), BPP sensor (right), Biopac MP36R (bottom) and Film Lab amplifier (top)

We have stacked aligned the BPP and PPG sensors (fig. 1) for the spatial-simultaneous acquisition of pressure and volume waveform. All signals are sampled at the $2 \mathrm{kHz}$ frequency with a resolution of 24-bit in Biopac acquisition unit. Sensor placement is shown in fig 3 below:

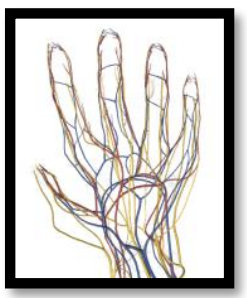

Figure 3: Sensor placement at index finger palmar artery

\section{Mathematical background}

Within the circulation system, the heart pumps out the blood in a non-continuous manner. The aorta is the biggest artery and acts as reservoir under the windkessel principle. Arterial compliance and peripheral resistance are directly affected the blood pressure waveforms which translates to PWV.

Moens-Korteweg equation formulates the PWV relationship in an elastic tube which is a useful tool to get insight into vascular diseases. The purpose of this study was to formulate the PU loop [1] and estimate the effect of overload in distal arteries which states that change in pressure is directly proportional to change in volume across the wavefront and describes as:

$$
\Delta p=C \rho \Delta v
$$

Where $\mathrm{C}$ is sonic velocity defined by Korteweg as:

$$
C=\sqrt{\frac{1}{\rho\left[\frac{1}{K}+\frac{D}{E e}\right]}}
$$

Where $\rho=$ Blood density, $K=$ Bulk modulus of fluid, $E=$ Young's modulus, the $\mathrm{e}=$ Wall thickness of the pipe and $\mathrm{D}=$ Artery diameter 


\section{Signal processing algorithm}

The data acquired in Biopac Acknowledge software is exported in excel format, for processing in MATLAB environment on an i7 Window PC. The algorithm [1] works in offline mode and processing sequence is described as below:

1- After pre-processing ECG signals R-peak is detected by the Pan-Tompkins method [11]

2- The Savitzky Golay filter is used to remove the noise and to take the difference of pressure and volume waveforms

3- Pressure and Volume waveform are ensembled by gating at R-peaks to remove signals outside of standard deviation of \pm 1 .

4- PU loop is formed by plotting difference of pressure again a difference of volume waveforms.

5- Normalized PU-loops and manually draw the slope between early systole (20-30\% of pressure values).

6- Multiply with viscosity to slope gives normalized PWV.

\section{Results - PU loop analysis}

The aforementioned signal processes algorithm was applied on the collated dataset and resulting plots for ensemble averages of differential pressure and volume waveforms are shown in figure $4 \& 5$. Manually drawn slope $20-30 \%$ systole give the local PWV as shown in fig 6 . and normalized PU Loop of all 6 subjects are plotted in fig 7. An increase PWV is shown in High BP subject was found to be 2.89 compared others.

Table 2: Dataset parameter and normalized PWV results

\begin{tabular}{|l|l|l|l|}
\hline BP start=>stop & Age & H(cm)/W(kg) BMI & PWV \\
\hline $139 / 98=>137 / 96$ & 45 & $175 / 85=>27.76$ & 2.89 \\
\hline $118 / 77=>125 / 77$ & 29 & $177 / 80=>25.54$ & 2.00 \\
\hline $116 / 71=>116 / 73$ & 32 & $174 / 78=>25.76$ & 1.75 \\
\hline $121 / 71=>127 / 72$ & 30 & $180 / 83=>25.62$ & 1.28 \\
\hline $119 / 66=>115 / 73$ & 27 & $188 / 73=>20.65$ & 1.11 \\
\hline $117 / 67=>122 / 68$ & 29 & $179 / 84=>26.22$ & 1.24 \\
\hline
\end{tabular}

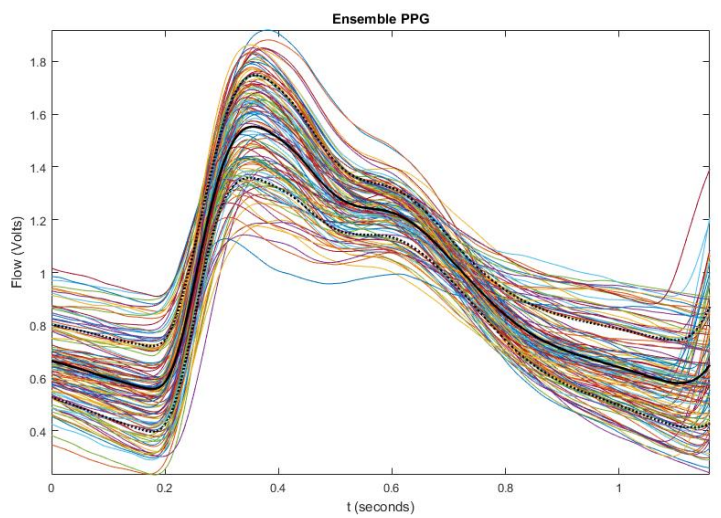

Figure 4: Ensembled PPG signals

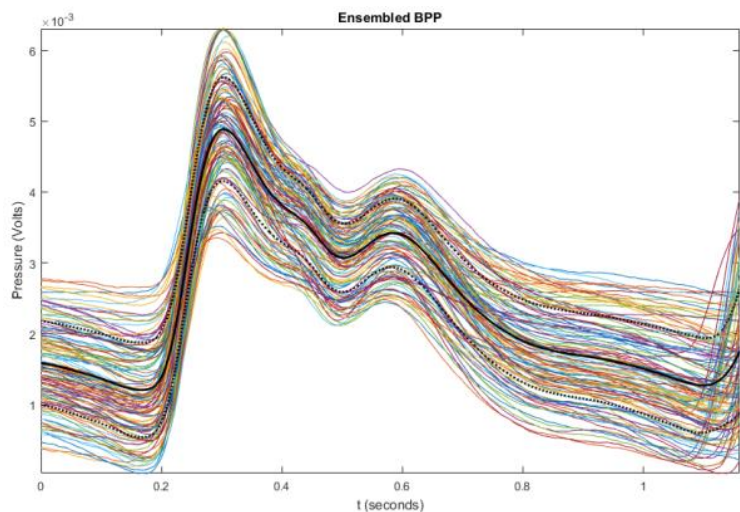

Figure 5: Ensembled BPP signals

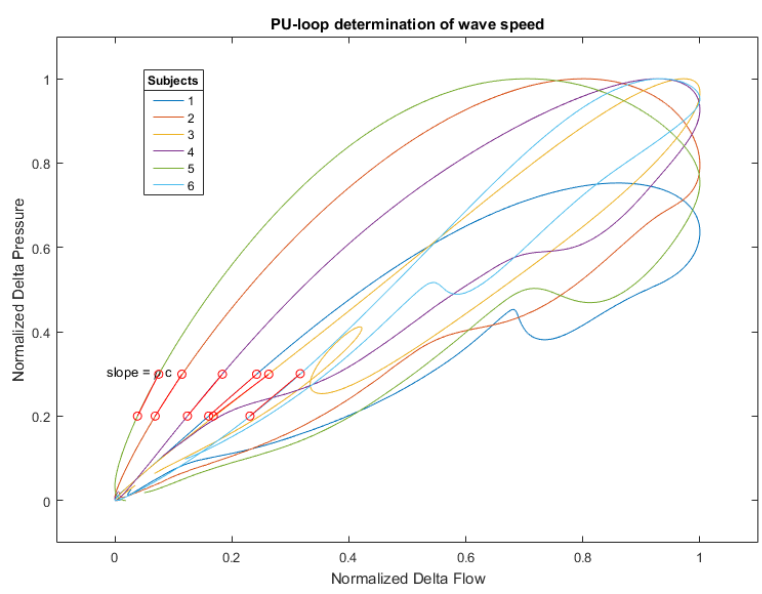

Figure 6: Morphology of Local PWV among subjects

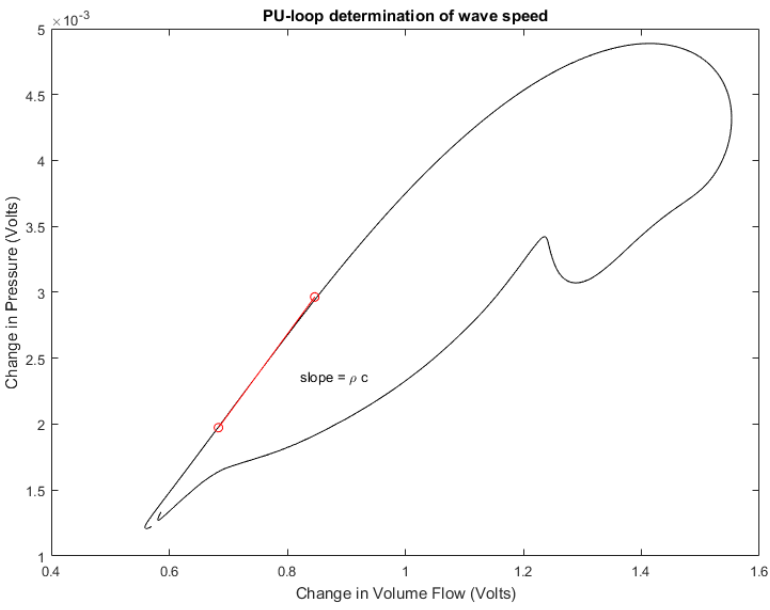

Figure 7: local PWV - Slope calculation

\section{Discussion}

The non-invasive assessment of BP using PTT techniques shows close co-relation to BP but lacks in tracking sudden changes in BP. The current methodology of Cuffless BP monitoring requires frequent input brachial $\mathrm{BP}$ to calibrate the measurement. This work has evaluated 
to contextualize the calibration requirement based on the steepness of PU loop for PTT estimation of BP. The analysis of non-invasive PU loop gives a valuable information on ventricular overload and afterload conditions.

The mandate of the study was to look at the feasibility of the formulation of PU loop for e-Health monitors. The quantization of pressure and volume flow waveform is required greater insight into biomechanics markers. In the current method used the normalization only to evaluates the morphology of the loop among the subjects and in future, it is planned to compare across hypertensive groups. The hydrostatic and gravitation effects are neglected in these experiments based on the assumption of the similar posture of the subjects, sitting on an armchair. The BPP sensor is very sensitive to the motion and subjects had to take account of this artifact. Scalability and repeatability of the experiment were very encouraging. This work is part of ongoing research for wearable design [12] to investigate the independence of correcting factor in Moens-Korteweg equation. These results were encouraging in finding local PWV which will be translated to the carotid artery by exploiting the symmetry of carotid bifurcation - to achieve virtual same location for acquisition site.

This method formulates the noninvasive assessment of PU loop which will be an assisting factor for PTT based Cuffless BP measurement techniques for e-Health industry. The PU loop assessment shows limitations at distal arteries arises due to degradation by higher reflection waves results in arteries bifurcation. In future, this method can be applied for hypertension a diagnosis.

\section{Conclusion}

In conclusion, a novel sensor system is designed for a non-invasive method for PU loop is an assessment in a palmar artery in index finger and results found symmetry in the morphology of PU loops among the healthy subjects and an increasing trend is found in PWV of high BP subjects.

\section{Acknowledgments}

This project has received funding from the European Union's Horizon 2020 research and innovation program under the Marie Sklodowska-Curie grant agreement No. 676201 .

\section{References}

[1] K. H. Parker, "An introduction to wave intensity analysis,” pp. 175-188, 2009.

[2] M. J. Roman, R. B. Devereux, J. R. Kizer, E. T. Lee, J. M. Galloway, T. Ali, J. G. Umans, and B. V. Howard, "Central pressure more strongly relates to vascular disease and outcome than does brachial pressure: The strong heart study," Hypertension, vol. 50, no. 1, pp. 197-203, 2007.

[3] G. P. J. Van Hout, R. De Jong, J. E. P. Vrijenhoek, L. Timmers, H. J. Duckers, and I. E. Hoefer, "Admittance-based pressure-volume loop measurements in a porcine model of chronic myocardial infarction," vol. 1, pp. 1565-1575, 2013.

[4] M. F. O'Rourke and D. E. Gallahger, "Pulse wave analysis," J. Hypertens., vol. 14, pp. 147-157, 1996.

[5] J. E. Davies, Z. I. Whinnett, D. P. Francis, K. Willson, R. A. Foale, I. S. Malik, A. D. Hughes, K. H. Parker, J. Mayet, E. Justin, Z. I. Whinnett, D. P. Francis, R. A. Foale, I. S. Malik, A. D. Hughes, H. Kim, and J. Mayet, "Use of simultaneous pressure and velocity measurements to estimate arterial wave speed at a single site in humans," pp. 59-61, 2006.

[6] B. W. A. M. M. Beulen, N. Bijnens, G. G. Koutsouridis, P. J. Brands, M. C. M. Rutten, and F. N. van de Vosse, "Toward Noninvasive Blood Pressure Assessment in Arteries by Using Ultrasound," Ultrasound Med. Biol., vol. 37, no. 5, pp. 788-797, 2011.

[7] M. A. Simon and M. R. Pinsky, "Right Ventricular Dysfunction and Failure in Chronic Pressure Overload," vol. 2011, 2011.

[8] R. Mukkamala, J.-O. Hahn, O. T. Inan, L. K. Mestha, C.-S. Kim, H. Töreyin, and S. Kyal, "Towards Ubiquitous Blood Pressure Monitoring via Pulse Transit Time: Theory and Practice," IEEE Trans Biomed Eng., vol. 62, no. 8, pp. 18791901, 2015.

[9] F. N. van de Vosse and N. Stergiopulos, "Pulse Wave Propagation in the Arterial Tree," Annu. Rev. Fluid Mech., vol. 43, no. 1, pp. 467-499, Jan. 2011.

[10] C. M. Mc Clean, J. Mc Laughlin, G. Burke, M. H. Murphy, T. Trinick, E. Duly, and G. W. Davison, "The effect of acute aerobic exercise on pulse wave velocity and oxidative stress following postprandial hypertriglyceridemia in healthy men," Eur. J. Appl. Physiol., vol. 100, no. 2, pp. 225-234, 2007.

[11] J. Pan and J. Willis, "A Real-Time QRS Detection Algorithm," vol. 1, no. 3, pp. 230-236, 1985.

[12] G. M. W. Janjua, P. J. Mclaughlin, D. Finlay, and D. Guldenring, "Wireless Chest Wearable Vital Sign Monitoring Platform," EMBC 2017.

Ghalib Muhammad Waqas Janjua

NIBEC, Ulster University, shore Road Newtownabbey, Co. Antrim Belfast United Kingdom BT37 0QB.

g.janjua@ulster.ac.uk 